\title{
The Contribution of Occupational Health Psychology to the Promotion of Well-Being at Work: an Evaluation of EAPS in the UK and China
}

\author{
Shan Liu ${ }^{1, a}$ \\ Shaanxi Xueqian Normal University, Xi'an, Shaanxi, China, 710100 \\ liushan0619@163.com
}

Keywords: Occupational Health Psychology, Well-Being, Eaps

\begin{abstract}
Employee assistance program (EAP) is a kind of interventions in workplace based on occupational health psychology (OHP). However, it was introduced to China only about ten years. The aim of this essay is to examine the contribution of OHP to the promotion of well-being at work though comparing the EAPs in the UK and China. The importance of promoting well-being at workis highlighted while the limitations and suggestions of it are also presented. To sum up, EAPs make great contributions to the promotion of well-being at work while the differences between that in the UK and China is significant. This may be of interest to people who want to further understand how OHP contribute to the well-being at work thought EAP and its implementation in different culture.
\end{abstract}

\section{Introduction}

Occupational health psychology (OHP) is a new research area focused on exploring how wellbeing at work is influenced in terms of psychological and physical dimensions. The concept of OHP is firstly raised by European psychologists (Barling \& Griffith, 2002). Since then, there are an increasing number of scholars and policymakers are dedicated to building healthy work organizations and developing the workplace interventions such as employee assistance program (EAP) though a better understanding of theories, models and assessment methods of OHP. EAP, as an important means of workplace intervention, has become fairly widely adopted by many organizations especially in Western countries for promoting their employees' well-being, reducing absenteeism and improving performance (Cooper, 2003).

Because of the world globalization, OHP as an independent discipline has spread to most of industrialized countries and even developing countries like China. In China the OHP only developed for about ten years and the EAPs are still very new for most of Chinese employees and domestic companies. Obviously, the gap between the UK and China in this field is huge. Almost all the paradigms and theories of Chinese EAPs model that of the UK and America regardless of culture issues in different contexts.

The aim of this essay is to analyzethe contribution of OHP to the promotion of well-being at work though comparing the EAPs in the UK and China. Firstly, there will be an overview of the importance of well-being at work and the way to promote it though EAPs. Then the development of EAPs in the UK and China will be examined respectively. Finally, comparison of the EAPs in the two countries will be discussed and cultural issues will be taken into consideration. The analysis of those may be helpful with deep understanding the importance of promoting workplace well-being though the development of EAPs in the UK and China. 


\section{The Importance of Well-Being at Work}

Working occupies employees' significant scale of their lives which are brought meanings and structured by it so that it is generally believed that employees should have a healthy and safe work context. Illness, injuries, and legislation should not be worried about. This belief has been approved by a number of developed countries such as the United States, the UK and even some developing countries including Indian and China (Kompier, 1996). In terms of the development of OHP, the focus has turned from workers' exposures to physical hazards in the work context into the promotion of well-being at work in a comfortable and appropriate work environment instead of the prevention of illness and injuries (Cooper \& Cartwright, 1994).

The theories of OHP could help to recognize the relationship between health and work and create effective interventions which could promote the employees' well-being, the performance and then in turn increase the productivity of the organization. Moreover, occupational health psychology is the base to address the problems in organizations such as absenteeism, turnover and work-related disorders. Because of the increasing morbidity of work-related disorders, interventions underpinned by OHP are requested in many countries as government regulations to manage the risk to psychological well-being, which in turn promote the development of occupational health psychology as an independent subject (Cox \& Griffiths, 2000).

In order to promote well-being at work, there are many models based on OHP to consider the management and prevention of stress. One of them is generally believed which have been identified interventions as primary, secondary, and tertiary levels. Primary prevention is referred to reducing or eliminating stressors and to promoting a healthyand supportivework context. Secondary prevention is referred tobreaking the link between the stressors and the consequent negative emotionssuch as anxiety and depression by changingboth individual and collective cognition of the stressors and improving stress coping skills. Tertiary prevention is about strategies that help employee rehabilitate and recover frompsychological or physical illness which is triggered by workrelated stress (Copper \& Cartwright, 1994). EAPs, as an important means of tertiary intervention, have become fairly widely adopted by many organizations especially in Western countries for promoting their employees' well-being, reducing absenteeismand improving performance effectiveness(Cooper, 2003).EAPs have been defined by the U.K. Employee Assistance Professionals Association (EAPA, 2009) as‘a worksite-based program designed to assist work organizations in addressing productivity issues and "employee clients" in identifying and resolving personal concerns including but not limited to, health, marital, family, financial, alcohol, drug, legal, emotional, or other personal issues that may affect job performance'.

\section{The History of EAP}

Employee Assistance Programmerstem from America. Primarily, EAP in America was established for financial loss attributed to alcohol abuse by a company named E. I. du Pont de Nemours in 1942. With the development ofoccupational health psychology underpinning the EAPs, theyturned to focus on addressing employees' individualand family concernsinstead of alcohol abuse (Roman, 1981). Whether the individual should be provided occupational health services no longer depended on the work performance but on the willingness of employees themselves. There are an increasing number of independentprofessional institutes offering occupational health services and interventions such as EAP counseling which are involved inthe employees'health insurance coverage in some developed countries (Steele, 1989). 
Because of some government legislations aimed at promotion the well-being at work and the development of occupational health psychology as anindependentdiscipline, there are an increasing number of kinds of occupational health service so as tomeet specific needs and situations. Most of companies in the UK and America try to identify the employees with work-related disordersand then refer them to specialist treatment institutes providing occupational health services, EAP as a significant means. Only some companies traintheir own staffas volunteers to help their fellows to be aware of and tackle their psychological problems. They usually undertake this program aftertheir daily work. However, there is only a few companies introducing the EAPs within their companies possibly because the limitation of time and resources essential for thesecarrying out.

\section{EAPs in the UK}

In the UK, the majority of employees' well-being and welfare could be guaranteed due to the establishment of the National Health Service in the 1940s.Until 1994, 5-6\% of the UK employees $(1,285,000)$ in 599 companieswere involved in EAPs (Cooper, 2003). However, it seems that the UK EAPs and short-term counseling industry is bigger than that of estimation. As one of the most significant occupational health interventions, it wassupposed that 1,695,000 Britishemployees from 775 companies would be covered at the increasing rate of 40\% after 1995 (Berridge, 1997).

The flourish of the industry of occupational health services is attributed to the impacts of workrelated stress which is an increasing economic concern for the UK employers. Nevertheless, one hand, because of the economic depression during the 1980s companies cut down the costs of welfare services, on the other hand, more productivity and better performance were requested which led to serious work-related stress in terms of alcoholism anddrug abuse, occupational maladjustment such aswork-family balance, absenteeism and psychological fatigue in the 1990s (Berridge, 1997).For instance, John Walker as asocial workerhas been compensated £175,000 forstress and legislationcosts successfully(Doherty \& Tyson, 1998). Moreover, Birmingham CityCouncil had to pay $£ 67,000$ foran employee as a result of work-related stress in July 1999.The Guardian newspaper has reported over 500 similar cases before these (Milne, 1999).

The British researchesabout alcohol and drug abuse EAPs have supported dramaticimprovement in work performance indicators such as decrease in absenteeism,complaint, accidents, turnover and lateness (Macdonald, 1997). Between 1970s and 1980s,the alcohol and drug abuse EAPs were compulsory when the symptoms were presented, the assumption perhapscould notbe extended to the current EAPs which was voluntary program to deal with different aspects of psychological problems such as work-family balance and burnout (Macdonald, 1997). According to McDonnell Douglas(Alexander Consulting Group, 1989), the study was independently carried out as a longitudinal research of expending more than 4 years before and after EAPs, involving a control group. It found that total saving was 4dollars with1 dollar spent (Berridge, 1997). It seems thatinterventions for drug and alcohol abusecould produce significant changes in job performance and absenteeism, but in the UK when the EAPs have been involved in the different health care system,the occupational health psychological models and theories that the EAPs would be based on and the aspects that occupational health services focus on both could influent the effectiveness of the EAPs while the evaluation and assessment should refer to the evaluation models of OHP.

In the 21st century it seems that UK National Health Service should not only focus on tertiary intervention but also the primary intervention which aim at preventing work-related stress. Only providing tertiary intervention such as EAPs in order tosave costs andimprove productivity may lead to disappointment. EAPs, as a method of stress management, could supportemployees who are experiencingwork-related disorders observably.However, from an integral perspective, primary, 
secondary and tertiary interventions as a whole theoretical OHP model to cope with work-related stress should be all take into consideration.

\section{EAPs in China}

With the development of economic and cultural globalization, EAPs and occupational health service agenciesspread to the developing countrieslike China, India and Brazil. There are now eight branches of EAP association in total in Central America, South America, Europe and Asia which is separated from the US based Employee Assistance Program Association (EAPA)(Masi, 1997). In China, with the rapid growth of economy in recent years, work-related stress and psychological disorders have become more and more common. Consequently, employees'wellbeing and the balance between organizations and individuals have been widely concernedby the public and the government. In thegovernment annual report of 2007,occupational health care and psychological counseling were suggested for promoting well-being at work while the positive management methods were recommended. However, the expenses of occupational health treatments are still not covered in the welfare system, which may limit the development of EAPs in China (Sun, 2011).

In the highly developed areas of China, the EAPs were introduced primarily by large international corporations and some government departments followingAmerica andthe UK emphasizing on expatriate problems.However, in some rural areas of China,because of the limitations of expertise resources and financial supports, organizations were unlikely to provide EAPs(Arthur, 2000). In terms of the techniques of EAPs based on occupational health psychology, it seems that there is only a few numbers of professionals in this field in China nowadays so thatdemand exceeds supply. As a result, there are an increasing number of colleges opened courses in this field but only a few of them could provide students intensive and professional trainings about EAPs while the market of occupational health services is still irregular and lack of agreed principles and monitoring from the government or the society. In general, it seems that not only the studies about the theories and assessments but also the application and standardized procedures of the EAPs are more prevalent in the UKthan that in China. Furthermore, the development of the EAPs in China is considerably influenced bywestern countries (Cooper, 2003).

\section{Compare and Contrast}

Along with the introduction of OHP as a branch of psychology from western countries to China, the awareness of the importance of physical and psychological health and safety at work is increased among both individuals and organizations. However, in China the OHP as an independent discipline only developed for about ten years and the EAPs are still very new for most of Chinese employees and domestic companies. Obviously, the gap between the UK and China in this field is huge. Almost all the paradigms and theories of Chinese EAPs copy that of the UK and America so that the effectiveness of it needs to be tested on the basis of OHP evaluation models in terms of the different cultural contexts.

In order to take OHP theories into practices and make EAPs suitable for Chinese market the cultural issues should be taken into consideration. Collectivism and individualism are two kinds of social types used to define cultural differences. China is a typical collectivistic country while the UK is individualistic(Arthur, 2000). Collectivism could be defined as a social typewhich ismade up by closely connected individuals who believe themselvesas a part of one or some collectives such as family and organizations and are driven by agreed beliefs, valuesand obligations.On the contrary, individualism may be defined as another social typewhichis made up by independent individuals 
who are driven by their own interests, needs and preferences. Organizations in collectivistic countries are more likely tooffer appropriatesocial supportswithin immediate work context instead of specific occupational health services agencies and EAPs which are popular in the UK. In fact, it seems that it tends to focus on social supports related positivity to cope with work-related stressin China contrast with the UK (Arthur, 2000).

To cope with work-related stress, both China and the UK provide their own cultural techniques. According to Lazarus (1995), in the UK individuals tend to use problem-focused coping style which is defined as an advanced attempt to change currentsituationswhile in China people have a tendency to emotion-focusedcoping strategies which is defined as an attempt to alter the disasters after related stress occurred. Culture seems tohave great influence on social support mechanisms.Both of the two adaptation techniquesassistemployees and their families coping with negativeeffects of work-related stress. Not onlyfinancial resources of the countries but also culturalstyles of dealing with stress areof significance. Emotion-focused coping is likely more emphasized than problem-focusedcoping so thatit may be difficult for the development of EAPs in this cultural context (Arthur, 2000).

Culture could have a significant impact on employee psychological processes underlying healthrelated outcomes, thus influencing how a healthy work environment could be built. From the macro prospective, how the occupational health services is organizes is affected by culture (Arthur, 2000) while culture also would shape employees' values and how they perceive and response to workrelated stress. In fact, as the economy becomes more diverse and as more organizations operate in a global context, the challenge for the OHP seems not only to better understand the psychological interventions contributing to employees' well-being but also to do so in a multicultural environment.

\section{Discussion}

Dealing with work-related problems is an increasingly significant part of the challenge of wellbeing at work settings. Most of the impacts of such issues seem to be mediated by work-related stress. It is supported that work-related stress is the main source of psychological disorders among employees. It is the responsibility for the government and the organization to reduce and prevent work-related stress. Occupational health psychology provides a framework for positive actions emphasizing interventions including EAPs.

The research reviews indicate that individual who hasserious psychological disorder could experience symptom reduction andfeel satisfied with the psychological counseling though EAPs provided by his employer, consequently, the absenteeismmight decrease. However, organizations may benefit less from the programin terms of productivity, performance morale and turnover which could produce profitable feedback fromEAPs investment (Hill, 2000). Therefore, based on these research findings, theHealth and Safety Commission (1999) suggests that EAPs are not tending to prevent work-related stress and reducing the possibility it happens. The objective is to teachindividuals how to deal with the effects of work-related stress when it has emerged.

With regard to the positive occupational health psychology (POHP), not only individuals who have experienced work-related disorders should received interventions but the normal employees also need positive interventions to reach a richer and more fulfilling existence (Seligman \& Csikszentmihalyi, 2000). Employs may be taught to use optimistic and even humor to cope with problems during working through positive EAPs. In this way, work engagement could be fostered so that absenteeism and turnover may be reduced while job performance may be improved, which contribute to employees' health and well-being. However, it is seems thatEAPs is only one of the variouspotential interventions for employers to deal with negative effects of work related stress.Taking sustainable actions to promote individual and organizational health and well-being 
requests a holistic strategy involving management, humanresource, occupational health and even cultural issues to evaluate principles, procedures and effectiveness.Specifically, muti-model interventions are needed instead of uni-model interventions. From an integrative perspective, both individual and organizational approaches as well as primary, second, tertiary and positive interventions should be taken into consideration as a whole based on occupational health psychology.

\section{Conclusion}

The purpose of this essay was to examine the contribution of OHP to the promotion of well-being at work. For this purpose,EAPs in the UK and Chinawere compared and contrasted based on OHP. The importance of well-being at work and the way to promote it though EAPs was presented. Furthermore, the development of EAPs in the UK and China was examined respectively. In addition, the EAPs in the two countries were compared and contrasted and cultural issues were taken into consideration.

To conclude, as a significant kind of interventions in OHP, EAPsmake great contributionsto the promotion of well-being at work. The present essaywasprovided for developing cross-cultural insights into the development of EAPs underpinned by OHP. EAPs areuseful to dealwith workrelated stressboth in the UK and China but its influence to the productivity, absenteeism and turnover seems not significant. However, the enhancing managerial health and effectiveness are beyondthis essay's concern. POHP were recommended to be involved in the EAPs while cultural difference should be taken into consideration during the implementation of EAPs. The analysis of those may be helpful with understanding the importance of promoting workplace well-being though the development of EAPs in the UK and China.

EAP is just one of various interventions, so that the contribution of OHP to promote well-being at work also needs to be analyzed from other perspectives. Moreover, consistent with the development of EAPs, this essay just drew on two countries: the UK and China. However, a various research paradigms, mixed methods and evaluation systemsbased on OHP in terms ofthe promotion of well-being at work should be explored in the future while a systematic analysis about the specific not only in the UK and China but also the world are requested.

\section{Reference}

1. Alexander Counseling Group (1989). Employee Assistance Program Financial Offset Study. Westport,CT: Alexander \& Alexander.

2. Arthur, A. R. (2000) 'Employee assistance programmes:the emperor's new clothes of stressmanagement’. British Journal of Guidance \& Counselling, 4(28), 549-559

3. Barling, J., and Griffiths, A. (2002). 'A history of occupational health psychology'. Handbook of occupational health psychology, 2(21), 19-33

4. Berridge, J., Cooper, C.L. and Highley, J.C. (1997). Employee Assistance Programmes andWorkplace Counselling. Chichester: Wiley.

5. Cooper, C.L., and Cartwright, S. (1994). 'Healthy mind, healthy organization: A proactive approach to occupational stress'. Human Ralations, 47(47), 455-471

6. Cooper, C.L., Dewe, P., and O’Driscoll, M. (2003). 'Employee Assistance program'. Handbook of work and health psychology, 18(21), 289-304 
7. Cox, T., Griffiths, A. a Rial-Gonzalez, E. (2000). Research on Work-Related Stress. Office for Official Publications of the European Communities: Luxembourg.

8. Doherty, N. and Tyson, S. (1998). Mental Well-being in the Workplace. Norwich: HMSO.

9. Employee Assistance Professionals Association. (2009). Standards of practice and professional guidelines for employee assistance programs. London: Author

10. Healthand Safety Commission (1999). Managing Stress at Work: a Discussion Document. London:Health and Safety Executive.

11. Highley, J.C. and Cooper, C.L. (1995). An assessment of employee assistance and workplace counselingprograms in British organisations. A report for the Health and Safety Executive. Unpublishedmanuscript.

12. Hill, C. (2000). 'The truth about workplace counseling'. Proceeding of the Occupational PsychologyConference of the British Psychological Society. 5(7), 99-103

13. Kompier, M.A.J. (1996). 'Job design and well-being'. Handbook of work and health psychology, 349-368

14. Lazrus, R.S. (1995). 'Psychological stress in the workplace’. Occupational Stress: a Handbook. 1(23), 3-14

15. Macdonald, S., Lothian, S. and Wells, S. (1997). 'Evaluation of an employee assistance program at atransportation company'. Evaluation and Program Planning, 20(23), 495-505

16. Masi, D. (1997). 'Evaluating employee assistance programs'. Research on Social Work Practice, 7(13),378-390

17. Milne, S. (1999). 'Rising stress brings desk rage at work'. The Guardian, 2(10), 25-38

18. Roman, P.M. (1981). 'From employee alcoholism to employee assistance: Analyses of thedeemphasize of preventionand alcoholism in work-based programs'. Journal of Studies on Alcohol, 42, 244-272.

19. Seligman, M., and Csikszentmihalyi, M. (2000). Positive Psychology: An introduction. American Psychologist, (2)55, 5-41

20. Steele, P.D. (1989). 'A History of Job-Based Alcoholism Programs'. Journal of Drug Issues, 19

(4), 511-532. Sun, Y.H. (2011). 'The development of EAP in China'. China Business and Trade, 2(5), 10-21 